\title{
Constraints on the Neutrino Flux in NOvA using the Near Detector Data
}

\author{
Kuldeep K. Maan*t \\ Fermilab/Panjab University, Chandigarh, India \\ E-mail: kuldeepmefnal.gov
}

\begin{abstract}
NOvA, a long-baseline neutrino oscillation experiment at Fermilab, is designed to measure electron-neutrino appearance and muon-neutrino disappearance in the NuMI beam. NOvA comprises of two finely segmented liquid scintillator detectors at 14 mrad off-axis in the NuMI beam. An accurate prediction of the neutrino flux is needed for precision oscillation and cross-section measurements. Data from the hadron-production experiments and, importantly, from the NOvA Near Detector provide powerful constraints on the muon-neutrino and electron-neutrino fluxes. In particular, the measurement of the neutrino-electron elastic scattering provides an in situ constraint on the absolute flux. This poster presents the data-driven predictions of the NOvA muonneutrino and electron-neutrino flux, and outlines future improvements in the flux determination.
\end{abstract}

38th International Conference on High Energy Physics

3-10 August 2016

Chicago, USA

\footnotetext{
${ }^{*}$ Speaker.

${ }^{\dagger}$ On behalf of the NOvA Collaboration
} 


\section{Introduction}

NOvA is a long-baseline accelerator neutrino oscillation experiment using the NuMI (Neutrinos at the Main Injector) $v_{\mu}$ beam. NOvA measures the rate of oscillations in two channels using a predominantly $v_{\mu}$ beam ( $v_{e}$ appearance $\& v_{\mu}$ disappearance). The NuMI beam line uses $120 \mathrm{GeV}$ protons beam from the Main Injector at Fermilab. These protons are then made collide against a graphite target [1]. The resulting hadrons are focused by two magnetic horns, and decay into neutrinos and other particles inside a long pipe. The neutrinos are observed by two detectors, Near (ND) and Far (FD) 14 mrad off-axis in the NuMI beam line. The ND is $100 \mathrm{~m}$ underground, $1 \mathrm{~km}$ from the source, used to measure composition of the unoscillated beam. The NOvA ND is 300-ton, low-Z, fine-grained ( 1 plane $0.15 \mathrm{X} 0$ ), highly active tracking calorimeter, optimized for EM shower reconstruction. In addition to oscillation study, the NOvA ND provides an excellent opportunity for the measurement of various neutrino interactions.
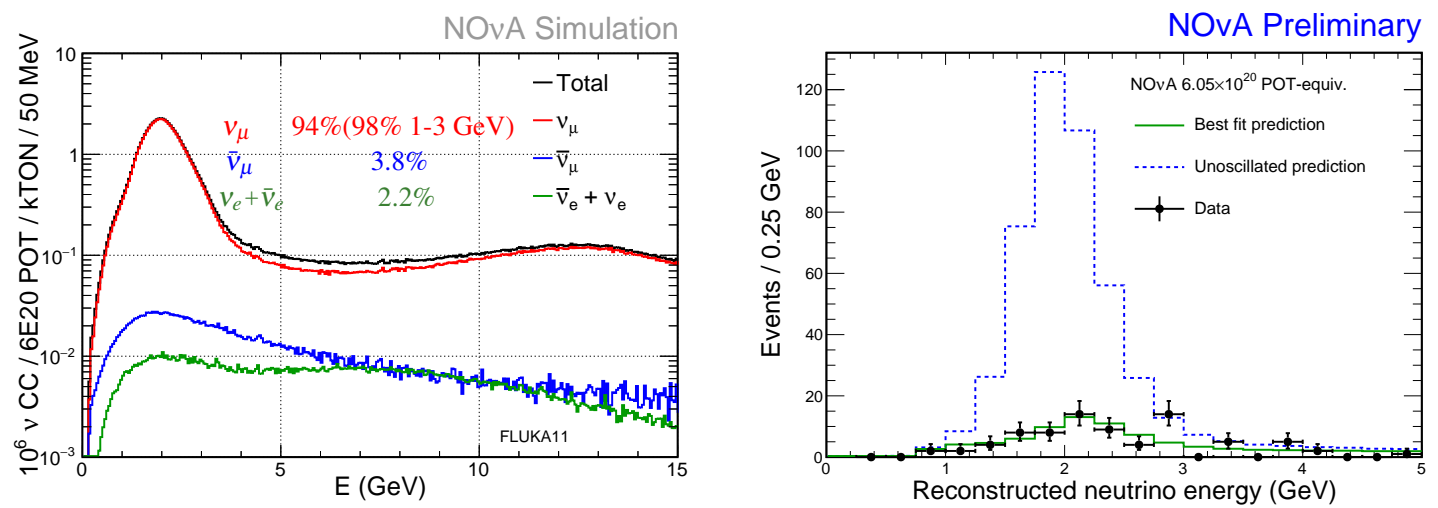

Figure 1: (Left) Neutrino flux at NOvA Near Detector with horns focus positive. (Right) The reconstructed energy distribution of $v_{\mu}$-CC events selected in the FD.

\section{Motivation}

The sensitivity of the oscillation measurements critically depends upon precise prediction of unoscillated $v_{\mu}, \bar{v}_{\mu}, v_{e}$ and $\bar{v}_{e}$ flux-ratio: $\frac{F D}{N D}\left(\mathrm{E}_{v}\right)$ (i.e. relative flux) as shown in Figure 1. The CP violation sensitivity is achieved by comparing the $v_{e}$ and $\bar{v}_{e}$ appearance rate in a FD. The intrinsic beam $v_{e}$ is a irreducible background for $v_{e}$ appearance analysis, and the flux is not fully cancelled between ND and FD. The absolute neutrino flux prediction is an essential ingredient to any cross section measurement $\sigma=\frac{N}{\Phi \cdot A \cdot \varepsilon}$ because it represents the normalization coefficient as a function of neutrino energy. The flux uncertainty goes into cross section uncertainty and vice versa. Dominant uncertainties in the neutrino flux come from poor knowledge of hadron production modeling. We need to constrain the flux using external hadron production data (MIPP [3], NA49 [2]). But at present the available external experiments data is mostly from thin targets for kaons as shown in Table 1 and able to constraint only 55\% flux in the higher energy region. Due to lack of kaons data from the hadron production experiments for thick carbon NuMI target we need to constrain the flux using NOvA ND Data. NOvA NuMI target is thick target. The fraction of neutrino flux 
from primary interactions is $55 \%$ but $45 \%$ comes from secondary and tertiary interactions, which is not covered by thin target external hadron-production experiments data as shown in Table. 1. At NOvA high energy neutrinos contribution is dominated by kaons but at present, there is no thick target hadron-production experiment data is available for kaons production. Therefore flux uncertainties are large in higher energy region as shown in Figure. 2 bottom (left).

\begin{tabular}{|c||c|c|c|c|c|}
\hline Experiment & Data type & $\mathrm{p}_{T}(\mathrm{GeV})$ & $\mathrm{x}_{F}$ & Target (Carbon) & Proton energy $(\mathrm{GeV})$ \\
\hline NA49 & $\pi$ production & $0-2$ & $0-0.5$ & thin & 158 \\
NA49 & K production & $0-1$ & $0-0.2$ & thin & 158 \\
MIPP & $\frac{K}{\pi}$ ratio & $0-2$ & $0.2-0.5$ & thin & 120 \\
MIPP & $\pi$ production & $0-2$ & $0-0.5$ & thick & 120 \\
\hline
\end{tabular}

Table 1: External data results from various experiments.
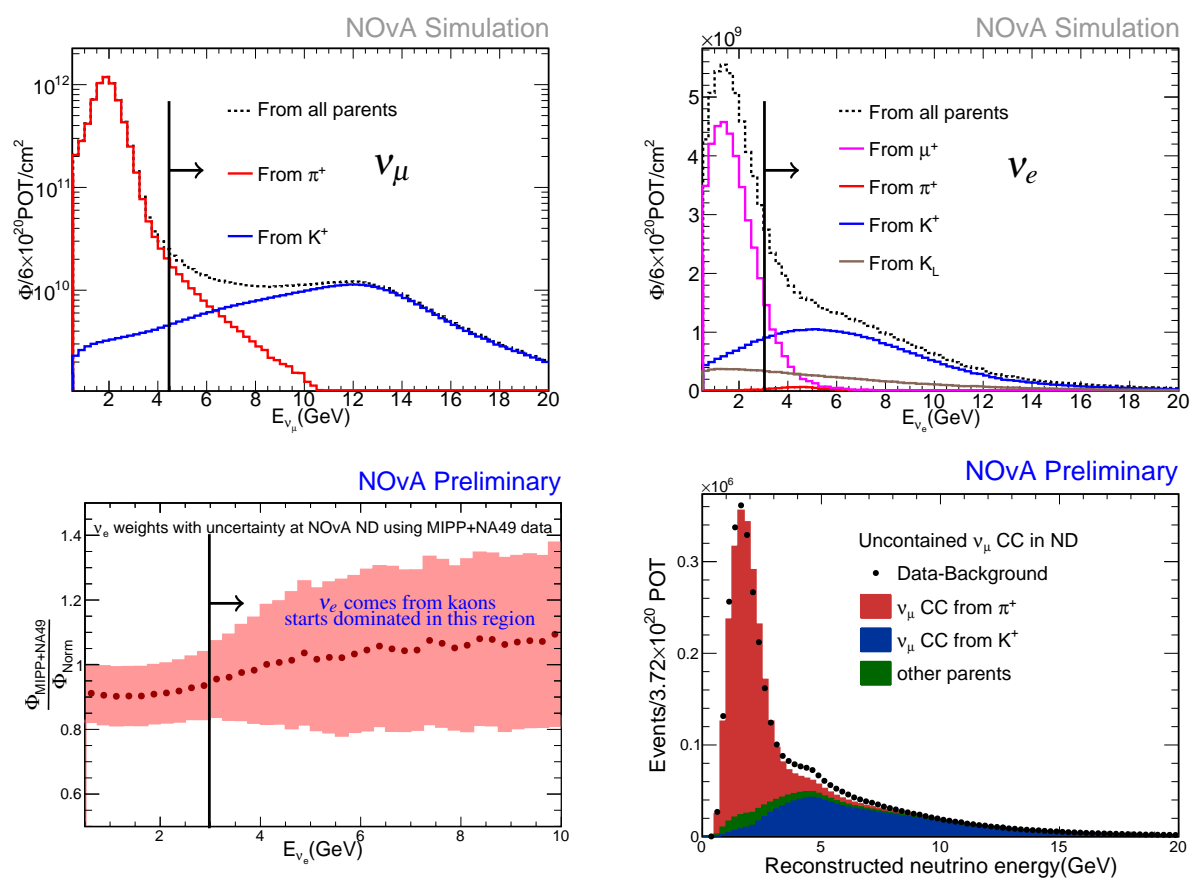

Figure 2: Top (left): NOvA $v_{\mu}$ flux distribution w.r.t parents information. Top (right): NOvA $v_{e}$ flux distribution w.r.t parents information. Bottom (left): Flux weight as a function of $E_{v_{e}}$ with uncertainty using MIPP+NA49 Data. Bottom (right): Uncontained $v_{\mu}$ CC events in ND.

\section{Data Driven Constraints using Near Detector $v_{\mu}$ CC Data}

The off-axis NuMI neutrino beam permits a clear separation between $v_{\mu}$ and $v_{e}$ induced from $K^{+}$and $\pi^{+}$for $E_{v_{\mu}} \geq 4.5 \mathrm{GeV}$ and $E_{v_{e}} \geq 3 \mathrm{GeV}$ as shown in Figure. 2 top (left, right). In the NOvA $\mathrm{ND}$, we are selecting 'uncontained' $v_{\mu}$-CC events (high- $E_{v}$ where the muon not ranges out in the detector) to obtained $K^{+}$yield using using $E_{v} \geq 4.5 \mathrm{GeV}$ as shown in Figure. 2 bottom (right). The analysis yields a $K^{+}$normalization of 1.17 relative to the FLUKA hadron-production model. The total systematic uncertainty in the $K^{+}$norm for $v_{\mu} \geq 4.5\left(v_{e} \geq 3 \mathrm{GeV}\right), \sim 8 \%$. 


\section{Data-Driven constraint on the total neutrino flux using $v$-e Elastic Scattering in the NOvA Near Detector}

The $v$-e elastic scattering is pure leptonic process which can be calculated accurately $(\sim 1 \%)$ and can be used to measure the absolute flux in NOvA ND. Due to small momentum transferred to the electron, signal is single forward electron in the final state. Unfortunately, the cross section $v$-e elastic scattering is suppressed relative to nucleon scattering by the ratio of the electron to nucleon masses and other kinematic factors, resulting in $\frac{\sigma_{v-e}}{\sigma_{v-N}} \sim 10^{-4}$. Therefore statistics are typically low in this channel. We are using two PID classifiers to identify $v$-e signal based on multilayer perceptron neural network. One of the PID distinguishes between electromagnetic showers and other backgrounds. Second PID specifically distinguishes between electron-induced and neutral pion induced showers by comparing $\frac{d E}{d x}$ in the first four planes. After selections on these variables, we employ a cut at $0.005 \mathrm{GeV} \times \mathrm{rad}^{2}$ on the kinematic variable $E_{e} \theta_{e}^{2}$, which is limited to very small values by the kinematics of the interaction itself as shown in Figure 3. The resulting electron energy spectrum, which will be used to constrain the flux, is shown in Figure. 3. It is expected that this technique will constrain the flux normalization to around $10 \%$ uncertainty. Work is in progress.
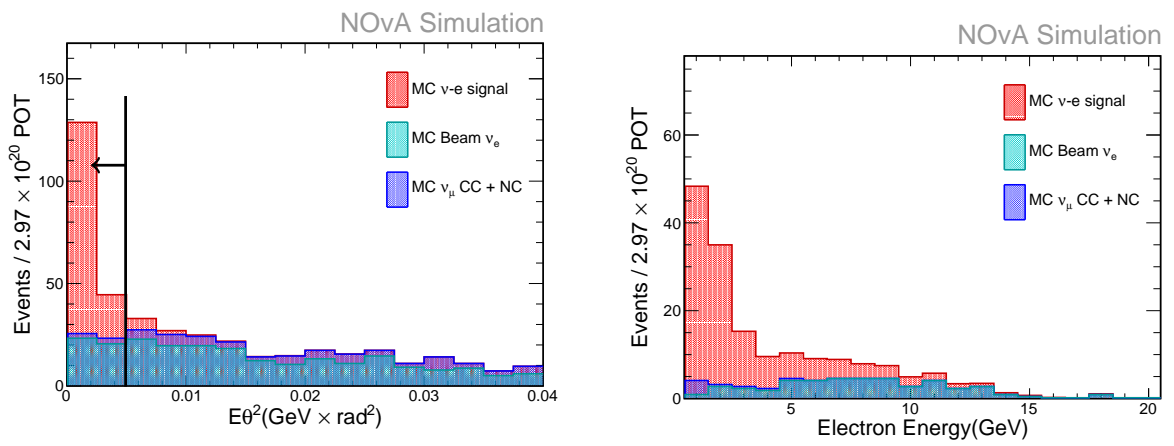

Figure 3: Electron $E_{e} \theta_{e}^{2}$ (left), where events with $E_{e} \theta_{e}^{2}<0.005 \mathrm{GeV} \times \mathrm{rad}^{2}$ are considered signal candidates, and resulting electron energy distribution (right) in the $v$-e elastic scattering analysis.

\section{Summary}

We expect to finalize the combine flux uncertainties from all these data-driven techniques soon.

\section{References}

[1] Adamson P et al. 2016 Nucl. Instrum. Meth. A806 279.

[2] NA49 Experiment, Eur. Phys. J. C 49 (2007), G. Tinti, Ph.D. Thesis, St. Cross College (2010).

[3] A. Lebedev, Ph.D. Thesis, Harvard University (2007), MIPP Experiment, Phys. Rev. D 90, 032001 (2014). 